\title{
A CONVERSE OF THE BOREL FORMULA
}

BY

RONALD M. DOTZEL

\begin{abstract}
When an elementary Abelian $p$-group acts on a $Z_{p}$-homology sphere ( $p$ a prime), it is known that the Borel formula must hold. Here we ask that the Borel formula hold and determine how this restricts, homologically, the type of space which can occur, assuming spherical fixed sets and connectedness. This is done by constructing a linear model of the action and an equivariant map to the model, the mapping cone of which yields certain homological information.
\end{abstract}

1. Introduction. If an elementary Abelian $p$-group $G, p$ a prime, acts on a $Z_{p}$-homology sphere $X$, it is well known that the Borel formula holds (see [1, p. 175]). Thus if $H \triangleleft G$, and $X^{H}$ is a $Z_{p}$-homology $n(H)$-sphere, one has

$$
n-n(G)=\sum(n(H)-n(G))
$$

where the sum runs over all subgroups $H$ of index $p$ (i.e. $H$ has corank 1 in $G)$.

We prove here the following converse.

THEOREM 1. Let $X$ be a finite $C W$-complex with a cellular action of $G=\left(Z_{p}\right)^{r}$, such that each $X^{H}(H \neq 0)$ is a $Z_{p}$-homology sphere. Suppose, moreover, that there exists an $n$ such that $\tilde{H}_{i}\left(X ; Z_{p}\right)=0, i \neq n$, and so that if $p$ is odd, $n-n(G)$ is even. Also assume the Borel formula holds for this action, i.e.

$$
n-n(G)=\sum(n(H)-n(G))
$$

where the sum runs over all corank 1 subgroups of $G$.

Then $H_{n}\left(X ; Z_{p}\right)=Z_{p} \oplus F$, with $F$ free over $Z_{p}[G]$.

REMARK. Assuming $n-n(G)$ even is a restriction only when $r=1$.

In $\$ 2$ we indicate how the theorem follows from the existence of a linear "model" of the action $S^{n}$, and an equivariant map $\phi: X \rightarrow S^{n}$ which induces $Z_{p}$ homology isomorphisms on all $X^{H}(H \neq 0)$, and is itself a $Z_{p}$ homology epimorphism.

In $\S 3$ we show that such a map $\phi$ exists and relegate the discussion of its homological properties to $\$ 4$.

Received by the editors December 7, 1977 and, in revised form, April 7, 1978.

AMS (MOS) subject classifications (1970). Primary 55C35; Secondary 55B25. 
This paper is the major part of my doctoral dissertation written at Rutgers University under G. E. Bredon. I wish to acknowledge my gratitude to him for suggesting this problem and for his generous advice and assistance.

2. We begin this section with the following lemma.

LEMMA 1. Let $X, G$ and $n$ be as in the statement of Theorem 1. Then there is a linear G-action on $S^{n}$ such that for $H \triangleleft G$, if $\left(S^{n}\right)^{H}=S^{m}$ then $m=n(H)$, where $X^{H}$ is a mod-p $n(H)$-sphere.

Proof. If $H$ is any corank 1 subgroup of $G$, the exact sequence

$$
0 \rightarrow H \rightarrow G \rightarrow G / H \rightarrow 0
$$

allows the formation of a 1-dimensional real nontrivial representation of $G$ if $p=2$, or complex nontrivial if $p \neq 2$, with $H$ acting trivially in either case. Let $\phi_{H}$ denote this representation.

For any representation $\psi, \psi^{m}$ denotes the representation $\psi \oplus \psi \oplus \ldots \oplus \psi$ ( $m$ times). 1 denotes the trivial real 1-dimensional representation. Consider, now, the real representations

or

$$
V=1^{n(G)}+\sum\left(\phi_{H}\right)^{n(H)-n(G)} \quad(\text { if } p=2)
$$

$$
V=1^{n(G)}+\sum\left(\phi_{H}\right)^{(n(H)-n(G)) / 2} \quad(\text { if } p \neq 2) .
$$

The sums are over all corank 1 subgroups $H$ of $G$. In either case

$$
\operatorname{dim}_{\mathbf{R}} V=n(G)+\sum(n(H)-n(G))=n
$$

with the sum as above. Letting $\alpha=2$ if $p \neq 2$ and $\alpha=1$ if $p=2$, one notes that for $K \triangleleft G$

$$
V^{K}=1^{n(G)}+\sum\left(\phi_{H}\right)^{(n(H)-n(G)) / \alpha} .
$$

This sum runs over $A_{K}=\{H \leqslant G \mid K \leqslant H, H$ corank 1 in $G\}$. Thus $\operatorname{dim}_{\mathrm{R}} V^{K}$ $=n(K)$. Compacting $V$ at one point yields the desired $S^{n}$.

From now on we will assume each of the spheres $S^{n(H)}$ is 1-connected and that $X$ is a suspended $G$-space (suspending $X$ is harmless homologically, and achieves the former condition).

We will complete this section by indicating how Theorem 1 follows from the existence of a $G$-map $\phi: X \rightarrow S^{n}$.

$\phi$ is expected to induce a $Z_{p}$-homology isomorphism on fixed sets for $H \neq 0$ and a $Z_{p}$-homology epimorphism for $H=0$.

We will need

LEMMA 2. Let $X$ be a finite $C W$-complex with an action of a p-group $P$, such that all $X^{H}(H<P, H \neq 0)$ are $Z_{p}$-acyclic. Suppose there is an integer $n>0$ so that $\tilde{H}_{i}\left(X ; Z_{p}\right)=0$ for $i \neq n$.

Then $H_{n}\left(X ; Z_{p}\right)$ is a free $Z_{p}[P]$-module. 
Proof. Let $\hat{X}=\cup\left\{X^{H} \mid 0 \neq H \leqslant P\right\}$. By Mayer-Vietoris and induction, $\hat{X}$ is $Z_{p}$-acyclic. Then $H_{n}\left(X / \hat{X} ; Z_{p}\right)=H_{n}\left(X ; Z_{p}\right) ; \bar{H}_{i}(X / \hat{X})$ consists of torsion prime to $p$ for $i \neq n$. Let $m(\geqslant n)$ be the cellular dimension of $X$.

Consider any surjection $f: Z[P] \rightarrow A$, where $A$ is a $Z[P]$-module of torsion prime to $p$, and $f$ is a $Z[P]$-homomorphism. It follows from $[4$, p. 236, 2.5] and $[6,4.12]$ that ker $f$ is $Z[P]$-projective (see [5, Lemma 3]).

Now by suspending, if necessary, we may assume $X / \hat{X}$ is 1-connected and so we may attach free orbits of cells to $X / \hat{X}$ to obtain a semifree $P$-space $Y$ with one fixed point. $Y$ is $m$-dimensional, $(n-1)$-connected and further, $H_{n}(Y)=H_{n}(X / \hat{X}) \oplus N$, where $N$ is a projective $Z[P]$-module. If $m=n$ then $Y$ satisfies the hypothesis of [5, Lemma 3] and so $H_{n}(Y)$ is $Z[P]$-projective, which implies that $H_{n}(X / \hat{X})$ is also $Z[P]$-projective.

If $m>n$, add free orbits of $(n+1)$-cells to kill off $N$. This produces a complex $X_{0}$ with $H_{n}\left(X_{0}\right)=H_{n}(X / \hat{X}), H_{n+1}\left(X_{0}\right)=H_{n+1}(X / \hat{X}) \oplus N_{0}$ (where $N_{0}$ is $Z[P]$-projective), and $H_{i}\left(X_{0}\right)=H_{i}(X / \hat{X})$ for $i>n+1$.

Add free orbits of $(n+1)$-cells to $X_{0}$ to kill off $H_{n}(X / \hat{X})$. This creates an $n$-connected complex (of dimension $m$ ) $Y_{n}$, so that $H_{i}\left(Y_{n}\right)=H_{i}(X / \hat{X})$, $i>n+1$; but we have

$$
0 \rightarrow H_{n+1}\left(X_{0}\right) \rightarrow H_{n+1}\left(Y_{n}\right) \rightarrow \operatorname{ker} \partial_{n+1} \rightarrow 0,
$$

where $\partial_{n+1}: H_{n+1}\left(Y_{n}, X_{0}\right) \rightarrow H_{n}\left(X_{0}\right)=H_{n}(X / \hat{X})$.

Now if $m=n+1$, it follows that $H_{n+1}\left(X_{0}\right)=N_{0}\left(\right.$ since $H_{n+1}\left(X ; Z_{p}\right)=0$ ) and since $Y_{n}$ is $(n+1)$-dimensional (this case), $n$-connected, by [5, Lemma 3], $H_{n+1}\left(Y_{n}\right)$ is $Z[P]$-projective. Therefore $H_{n}(X / \hat{X})$ has finite projective dimension and by $[6,4.12]$, actually has projective dimension $<1$.

Now if $m>n+1$, step 3 is as follows. To $Y_{n}$, add free orbits of $(n+2)$ cells to kill off $H_{n+1}\left(X_{0}\right)$ (see sequence (*)). This produces a complex $Y_{n+1}^{\prime}$ with $H_{n+1}\left(Y_{n+1}^{\prime}\right)=\operatorname{ker} \partial_{n+1}$ and $H_{n+2}\left(Y_{n+1}^{\prime}\right)=H_{n+2}\left(X_{0}\right) \oplus N_{1}$, where $N_{1}$ is $Z[P]$-projective.

Now add free orbits of $(n+2)$-cells to $Y_{n+1}^{\prime}$ to form the complex $Y_{n+1}$ and to kill off ker $\partial_{n+1}$. Note that $Y_{n+1}$ is $(n+1)$-connected, m-dimensional. Furthermore we have the exact sequence

$$
H_{n+2}\left(Y_{n+1}, Y_{n+1}^{\prime}\right) \rightarrow H_{n+1}\left(Y_{n+1}^{\prime}, X_{0}\right) \stackrel{\partial_{n+1}}{\rightarrow} H_{n}\left(X_{0}\right)=H_{n}(X / \hat{X}),
$$

where the left-hand map is the composition

$$
H_{n+2}\left(Y_{n+1}, Y_{n+1}^{\prime}\right) \stackrel{\partial_{n+2}}{\rightarrow} H_{n+1}\left(Y_{n+1}^{\prime}\right) \approx \operatorname{ker} \partial_{n+1} \mapsto H_{n+1}\left(Y_{n+1}^{\prime}, X_{0}\right) .
$$

We also have the exact sequence

$$
0 \rightarrow H_{n+2}\left(Y_{n+1}^{\prime}\right) \rightarrow H_{n+2}\left(Y_{n+1}\right) \rightarrow H_{n+2}\left(Y_{n+1}, Y_{n+1}^{\prime}\right) \stackrel{\partial_{n+2}}{\rightarrow} H_{n+1}\left(Y_{n+1}^{\prime}\right) \text {. }
$$

Now if $m=n+2$, it follows that $H_{n+2}\left(Y_{n+1}^{\prime}\right)=N_{1}$, a $Z[P]$-projective, because $H_{n+2}\left(X ; Z_{p}\right)=0$. Since $Y_{n+1}$ is, in this case, $(n+2)$-dimensional, 
and $(n+1)$-connected, $H_{n+2}\left(Y_{n+1}\right)$ is $Z[P]$-projective by [5, Lemma 3]. Thus $H_{n}(X / \hat{X})$ has finite projective dimension (simply link the sequences together). By [6,4.12], it has projective dimension $\leqslant 1$.

If $m>n+2$, then we have the exact sequence

$$
0 \rightarrow H_{n+2}\left(Y_{n+1}^{\prime}\right) \rightarrow H_{n+2}\left(Y_{n+1}\right) \rightarrow \operatorname{ker} \partial_{n+2} .
$$

Since $H_{n+2}\left(Y_{n+1}^{\prime}\right)=H_{n+2}\left(X_{0}\right) \oplus N_{1}$, step 3 may be repeated until $Y_{m-1}$ is reached. The same argument, above, shows that $H_{n}(X / \hat{X})$ has finite projective dimension over $Z[P]$, and hence has projective dimension $<1$.

So, for suitable projectives $R, S$ over $Z[P]$,

$$
0 \rightarrow R \rightarrow S \rightarrow H_{n}(X / \hat{X}) \rightarrow 0
$$

is an exact sequence. Since $H_{n}(X / \hat{X})$ has no $p$-torsion,

$$
0 \rightarrow R \otimes_{Z} Z_{p} \rightarrow S \otimes_{Z} Z_{p} \rightarrow H_{n}\left(X / \hat{X} ; Z_{p}\right) \rightarrow 0
$$

is exact over $Z_{p}[P]$. By [6,3.5], $R \otimes_{Z} Z_{p}$ is injective (as well as free) and so $H_{n}\left(X / \hat{X} ; Z_{p}\right)$, a summand of the free $Z_{p}[P]$ module $S \otimes_{Z} Z_{p}$, is free by [6,3.5]. Since $H_{n}\left(X / \hat{X} ; Z_{p}\right)=H_{n}\left(X ; Z_{p}\right), H_{n}\left(X ; Z_{p}\right)$ is free.

LemMA 3. Given $G, X, S^{n}$ as above. Suppose $\phi: X \rightarrow S^{n}$ is a G-map which induces a $Z_{p}$-homology isomorphism $\phi^{H}$ for $H \neq 0$ and an epimorphism for $H=0$, where $\phi^{H}: X^{H} \rightarrow\left(S^{n}\right)^{H}$.

Then $H_{n}\left(X ; Z_{p}\right)=Z_{p} \oplus F$ where $F$ is a free $Z_{p}[G]$ module.

Proof. $C_{\phi}$, the mapping cone of $\phi$, inherits a $G$-action and $\left(C_{\phi}\right)^{H}=C_{\phi^{H}}$ for $H \leqslant G$. Thus each $\left(C_{\phi}\right)^{H}$ is $Z_{p}$-acyclic.

A Mayer-Vietoris argument on $C_{\phi}$ produces

$$
\ldots \rightarrow H_{i}(X) \stackrel{\phi_{*}}{\rightarrow} H_{i}\left(S^{n}\right) \rightarrow H_{i}\left(C_{\phi}\right) \rightarrow H_{i-1}(X) \rightarrow \ldots,
$$

$Z_{p}$-coefficients throughout. Now $\tilde{H}_{i}\left(C_{\phi} ; Z_{p}\right)=0, i \neq n+1$, and so by Lemma $2, H_{n+1}\left(C_{\phi} ; Z_{p}\right)$ is $Z_{p}[P]$ free, hence also injective. Consequently

$$
0 \rightarrow H_{n+1}\left(C_{\phi} ; Z_{p}\right) \rightarrow H_{n}\left(X ; Z_{p}\right) \rightarrow H_{n}\left(S^{n} ; Z_{p}\right) \rightarrow 0
$$

splits over $Z_{p}[P]$.

3. We now will show that the equivariant map mentioned in Lemma 3 exists. In order to do this we use the equivariant obstruction theory of Bredon [3, Chapter 2] to show that the map constructed in the following lemma may be extended equivariantly.

LEMMA 4. If $G, X, S^{n}$ are as above, then there is a map $\phi: X^{G} \rightarrow S^{G}$ inducing a $Z_{p}$-homology isomorphism.

Proof. Let $K\left[Z_{p}, n(G)\right]$ be an Eilenberg-Mac Lane complex whose $n(G)$ skeleton is $S^{n(G)}$. Corresponding to a generator of $H^{n(G)}\left(X^{G} ; Z_{p}\right) \approx Z_{p}$ is a 
cellular map $\phi: X^{G} \rightarrow K\left[Z_{p}, n(G)\right]$. From the commutative diagram (vertical maps are inclusions)

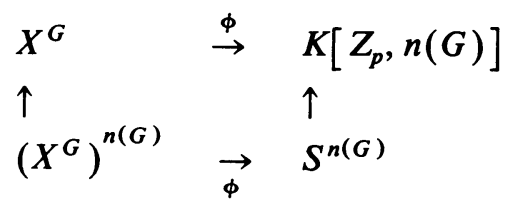

it follows that $\phi:\left(X^{G}\right)^{n(G)} \rightarrow S^{n(G)}$, the restriction of $\phi$ to the $n(G)$-skeleton is a $Z_{p}$-homology epimorphism. By classical obstruction theory and a Universal Coefficient Theorem one finds that any obstructions to extending $\phi$ to all of $X^{G}$ have finite order, prime to $p$. This is a minor impediment; for, suppose $\phi$ : $\left(X^{G}\right)^{(m)} \rightarrow S^{G}$ and an obstruction to extending $\phi$ to $\left(X^{G}\right)^{(m+1)},\left[C_{\phi}\right]$, has order $k$, with $p \nmid k$. Replace $\phi$ by the map

$$
\psi:\left(X^{G}\right)^{(m)} \rightarrow\left(X^{G}\right)^{(m)} \vee \cdots \vee\left(X^{G}\right)^{(m)} \rightarrow S^{G} \vee \cdots \vee S^{G} \rightarrow S^{G}
$$

where the second and third terms are $k$-fold wedges and the middle map is $k$-copies of $\phi$. Since we are assuming $X$ is a suspension, so are all its fixed sets; and then the first map is cogroup addition while the last is a collapse. By homotopy addition one checks that the obstruction to extending $\psi$ (corresponding to $C_{\phi}$ ), namely $C_{\psi}$, is $k C_{\phi}=0$. Also, in homology with $Z_{p}$ coefficients, $\psi_{*}=u \phi_{*}$, where $u$ is a unit in $Z_{p}$, so $\psi_{*}$ will also be an epimorphism. Notice that once $\phi$ is extended, $\phi_{*}$ will be an isomorphism.

Now suppose we have an equivariant map $\phi: X^{(k)} \cup X^{G} \rightarrow S^{n}$. Obstructions to extending $\phi$ equivariantly to $X^{(k+1)}$ lie in $H_{G}^{k+1}\left(X, X^{G}\right.$; $\tilde{\omega}_{k}\left(S^{n}\right)$ ), the equivariant classical cohomology group with coefficient system $\tilde{\omega}_{k}\left(S^{n}\right)$ (see Bredon [3, Chapter 2]). Because all of the $S^{H}$ are 1-connected, we can assume $\phi$ is extended to $\phi: X^{(2)} \cup X^{G} \rightarrow S^{n}$.

The same argument as in Lemma 4 shows that if the obstruction, [ $C_{\phi}$ ], in $H_{G}^{k+1}\left(X, X^{G} ; \tilde{\omega}_{k}\left(S^{n}\right)\right)$ has finite order prime to $p$, we may replace the $G$-map $\phi$ by the $G$-map $\psi$. $\psi$ will have the same $Z_{p}$-homological properties, and $\left[C_{\psi}\right]=0$, as before.

The remainder of this section will be devoted to showing that the groups $H_{G}^{k+1}\left(X, X^{G} ; \tilde{\omega}_{k}\left(S^{n}\right)\right)$ have finite order, prime to $p$, or are zero. This done, $\phi$ can be extended.

We first consider a special case.

LEMMA 5. Let $X$ be a finite $C W$-complex with the $Z_{p}$-homology of an $n$-sphere. Suppose $G=Z_{p}$ acts cellularly on $X$. Let $\phi: X^{G} \rightarrow\left(S^{n}\right)^{G}$ induce a $Z_{p}$-homology isomorphism. Assume (without loss) that $n(G)=\operatorname{dim}\left(S^{n}\right)^{G}>2$. Then all of the obstruction groups $H_{G}^{k+1}\left(X, X^{G} ; \tilde{\omega}_{k}\left(S^{n}\right)\right)$ are of finite order, prime to $p$, or zero. 
Proof. Assume $\phi$ has been extended to $X^{(r-1)} \cup X^{G}$, equivariantly. Obstructions to extending $\phi$ to $X^{(r)} \cup X^{G}$ are represented by cochains residing in

$$
C_{G}^{r}\left(X, X^{G} ; \tilde{\omega}_{r-1}\left(S^{n}\right)\right) \approx \operatorname{Hom}\left(C_{r}\left(X, X^{G} ; Z\right), \tilde{\omega}_{r-1}\left(S^{n}\right)\right) .
$$

(Hom used here refers to natural transformations between the two contravariant functors $C_{r}\left(X, X^{G} ; Z\right)$ (see $[3$, p. I.20] for the definition) and $\tilde{\omega}_{r-1}\left(S^{n}\right)$; the isomorphism is a chain isomorphism (also see [3, p. I.9]). All such transformations form an Abelian group [3, p. I.9].)

Now for any $r$-cell $\sigma$, not in $X^{G}, G_{\sigma}$, the isotropy subgroup of $G$, is 0 and so

$$
C_{r}\left(X, X^{G} ; Z\right)\left(G / G_{\sigma}\right)=C_{r}\left(X, X^{G} ; Z\right) .
$$

Also, $\tilde{\omega}_{r-1}\left(S^{n}\right)\left(G / G_{o}\right)=\pi_{r-1}\left(S^{n}\right)$. If $r \leqslant n$, it follows that

$$
\operatorname{Hom}\left(C_{r}\left(X, X^{G} ; Z\right) ; \tilde{\omega}_{r-1}\left(S^{n}\right)\right) \approx \operatorname{Hom}_{Z G}\left(C_{r}\left(X, X^{G} ; Z\right), \pi_{r-1}\left(S^{n}\right)\right)=0 \text {, }
$$

so we may assume $\phi: X^{(n)} \cup X^{G} \rightarrow S^{n}$ is an equivariant extension of $\phi$ : $X^{G} \rightarrow S^{G}$.

Obstructions to extending $\phi$ to $X^{(n+1)}$ are represented by cochains in $\operatorname{Hom}\left(C_{n+1}\left(X, X^{G}\right), \tilde{\omega}_{n}\left(S^{n}\right)\right)$. Each element of this group is a diagram

$$
\begin{array}{cll}
C_{n+1}\left(X, X^{G}\right) & \rightarrow & \pi_{n}\left(S^{n}\right) \\
\uparrow & & \uparrow \\
0 & \rightarrow & \pi_{n}\left(\left(S^{n}\right)^{G}\right)
\end{array}
$$

where the horizontal maps are $Z G$-homomorphisms. Thus

$$
\operatorname{Hom}\left(C_{n+1}\left(X, X^{G}\right), \tilde{\omega}_{n}\left(S^{n}\right)\right) \approx \operatorname{Hom}_{Z G}\left(C_{n+1}\left(X, X^{G} ; Z\right), \pi_{n}\left(S^{n}\right)\right),
$$

and also

$$
H_{G}^{n+1}\left(X, X^{G} ; \tilde{\omega}\left(S^{n}\right)\right) \approx H^{n+1}\left(\operatorname{Hom}_{Z G}\left(C_{*}\left(X, X^{G} ; Z\right), \pi_{n}\left(S^{n}\right)\right)\right) .
$$

Now consider $\operatorname{Hom}_{Z G}\left(C_{*}\left(X, X^{G} ; Z\right), Z_{p}\right)$, which by $[4$, p. 30, 3'] (and using that $C_{*}\left(X, X^{G} ; Z\right)$ is a free $Z G$ complex $)$ is chain isomorphic to $\operatorname{Hom}_{Z_{p} G}\left(C_{*}\left(X, X^{G} ; Z_{p}\right), Z_{p}\right)$.

If $m$ is the cellular dimension of $X$ and $B_{n}\left(X, X^{G} ; Z_{p}\right)$ the relative $n$-boundaries $\bmod p$, then the sequence

$$
0 \rightarrow C_{m}\left(X, X^{G} ; Z_{p}\right) \rightarrow \cdots \rightarrow C_{n+1}\left(X, X^{G} ; Z_{p}\right) \rightarrow B_{n}\left(X, X^{G} ; Z_{p}\right) \rightarrow 0
$$

is exact because $H_{i}\left(X, X^{G} ; Z_{p}\right)=0$ if $i>n$. Thus $B_{n}\left(X, X^{G} ; Z_{p}\right)$ has finite projective dimension over $Z_{p} G$ and by $[6,3.5], B_{n}\left(X, X^{G} ; Z_{p}\right)$ is projective. Thus for any $k \geqslant 2$,

$$
H^{n+k}\left(\operatorname{Hom}_{Z_{p} G}\left(C_{*}\left(X, X^{G} ; Z_{p}\right), Z_{p}\right)\right)=\operatorname{Ext}_{Z_{p} G}^{k-1}\left(B_{n}\left(X, X^{G} ; Z_{p}\right), Z_{p}\right)=0 \text {. }
$$


Using that $B_{n}\left(X, X^{G} ; Z_{p}\right)$ is $Z_{p} G$ injective, one also has

$$
H^{n+1}\left(\operatorname{Hom}_{Z_{p} G}\left(C_{*}\left(X, X^{G} ; Z_{p}\right), Z_{p}\right)\right)=0 .
$$

Corresponding to the coefficient sequence

$$
0 \rightarrow Z \stackrel{p}{\rightarrow} Z \rightarrow Z_{p} \rightarrow 0
$$

there is the long exact sequence

$$
\begin{aligned}
\ldots & \rightarrow H^{n+k}\left(\operatorname{Hom}_{Z G}\left(C_{*}\left(X, X^{G} ; Z\right), Z\right)\right) \\
& \stackrel{p}{\rightarrow} H^{n+k}\left(\operatorname{Hom}_{Z G}\left(C_{*}\left(X, X^{G} ; Z\right), Z\right)\right) \rightarrow 0
\end{aligned}
$$

for $k>1$. Since $H^{n+k}\left(\operatorname{Hom}_{Z G}\left(C_{*}\left(X, X^{G} ; Z\right), Z_{p}\right)\right)=0$, it follows that

$$
H^{n+k}\left(\operatorname{Hom}_{Z G}\left(C_{*}\left(X, X^{G} ; Z\right), Z\right)\right), \quad k>1,
$$

has torsion prime to $p$ (if $p=2, Z$ may have a nontrivial action).

In order to examine $H_{G}^{n+k}\left(X, X^{G} ; \tilde{\omega}_{n+k-1}\left(S^{n}\right)\right)$ for $k>2$ we first note, as above, that it is isomorphic to $H^{n+k}\left(\operatorname{Hom}_{Z G}\left(C_{*}\left(X, X^{G} ; Z\right), \pi_{n+k-1}\left(S^{n}\right)\right)\right)$. Since if $\pi_{n+k-1}\left(S^{n}\right)$ is infinite it must have form $Z \oplus A$ ( $A$ finite) by [8, p. 516], it follows from the sequence of $Z G$-modules

$$
0 \rightarrow A \rightarrow Z \oplus A \rightarrow Z \rightarrow 0
$$

that it suffices to consider only $H^{n+k}\left(\operatorname{Hom}_{Z G}\left(C_{*}\left(X, X^{G} ; Z\right), A\right)\right)$, where $A$ is a finite $Z G$-module. Moreover it is quite clear that one need only consider $H^{n+k}\left(\operatorname{Hom}_{Z G}\left(C_{*}\left(X, X^{G} ; Z\right), P\right)\right)$ where $P$ is the $p$-sylow subgroup of $A$. Thus whether or not $H^{n+k}\left(\operatorname{Hom}_{Z G}\left(C_{*}\left(X, X^{G} ; Z\right), \pi_{n+k-1}\left(S^{n}\right)\right)\right)$ is torsion prime to $p$ depends entirely on the behavior of $H^{n+k}\left(\operatorname{Hom}_{Z G}\left(C_{*}\left(X, X^{G} ; Z\right)\right.\right.$, $P$ )). In case $P=Z_{p}$, from above, this latter group is 0 . If $P$ is a vector space over $Z_{p}$, by [7, p. 80] the automorphism $\alpha$ on $P$ induced by the $G$ action has a proper invariant subgroup. If $P$ is not a $Z_{p}$-vector space then the proper subgroup $V(P)$ of all elements of order $p$ in $P$ is an invariant subgroup. In any case then, let $H$ be some invariant subgroup of $P$. Using the Bockstein sequence from

$$
0 \rightarrow H \rightarrow P \rightarrow P / H \rightarrow 0
$$

and induction on the order of $P$, the conclusion that

$$
H^{n+k}\left(\operatorname{Hom}_{Z G}\left(C_{*}\left(X, X^{G} ; Z\right), P\right)\right)=0
$$

follows. So $H^{n+k}\left(\operatorname{Hom}_{Z G}\left(C_{*}\left(X, X^{G} ; Z\right), \pi_{n+k-1}\left(S^{n}\right)\right)\right)$ is torsion prime to $p$, and this proves the lemma.

Now the general problem of extending $\phi: X^{(2)} \cup X^{G} \rightarrow S^{n}$ equivariantly, with $G=Z_{p}^{r}$, brings us to possible obstructions lying in $H_{G}^{k+1}\left(X, X^{G}\right.$; $\left.\tilde{\omega}_{k}\left(S^{n}\right)\right)$ and, as remarked above, it suffices to show that these groups have finite order prime to $p$. 
Now for convenience, if $A$ is an abelian group, let $A_{p}$ and $p A$ denote the elements of order $p$ in $A$ and the image of multiplication by $p$, respectively. We want to define several contravariant coefficient systems. Contravariant coefficient systems are contravariant functors on the category of coset spaces $G / H$ and equivariant maps (with respect to left translation) $G / H \rightarrow G / K$. These are $\left(\tilde{\omega}_{k}\left(S^{n}\right)\right)_{p}, p \tilde{\omega}_{k}\left(S^{n}\right)$ and $\tilde{\omega}_{k}\left(S^{n}\right) \otimes Z_{p}$, defined on objects $G / H$ by $\left(\tilde{\omega}_{k}\left(S^{n}\right)\right)_{p}(G / H)=\left(\pi_{k}\left(S^{H}\right)\right)_{p}, p \tilde{\omega}_{k}\left(S^{n}\right)(G / H)=p \pi_{k}\left(S^{H}\right)$ and $\left(\tilde{\omega}_{k}\left(S^{n}\right) \otimes\right.$ $\left.Z_{p}\right)(G / H)=\pi_{k}\left(S^{H}\right) \otimes Z_{p}$ respectively, and obvious values on morphisms. It is easy to check that these are contravariant coefficient systems. The following lemma is routine.

LEMMA 6. There are two short exact sequences in $C_{G}$ (the Abelian category of contravariant coefficient systems):

$$
\begin{aligned}
& 0 \rightarrow\left(\tilde{\omega}_{k}\left(S^{n}\right)\right)_{p} \stackrel{j}{\rightarrow} \tilde{\omega}_{k}\left(S^{n}\right) \stackrel{p}{\rightarrow} p \tilde{\omega}_{k}\left(S^{n}\right) \rightarrow 0, \\
& 0 \rightarrow p \tilde{\omega}_{k}\left(S^{n}\right) \stackrel{i}{\rightarrow} \tilde{\omega}_{k}\left(S^{n}\right) \stackrel{\pi}{\rightarrow} \tilde{\omega}_{k}\left(S^{n}\right) \otimes Z_{p} \rightarrow 0 .
\end{aligned}
$$

REMARK. $i$ and $j$ are "inclusion" natural transformations, and $\pi$ is defined by the commutative (for $G / H \stackrel{f}{\rightarrow} G / K$ equivariant) diagram and the desire that $\pi$ is not (generally) zero:

$$
\begin{array}{lll}
\pi_{k}\left(S^{H}\right) & \rightarrow & \pi_{k}\left(S^{H}\right) \otimes Z_{p} \\
\uparrow \tilde{\omega}_{k}(f) & & \uparrow \tilde{\omega}_{k}(f) \otimes 1 \\
\pi_{k}\left(S^{K}\right) & \rightarrow & \pi_{k}\left(S^{K}\right) \otimes Z_{p}
\end{array}
$$

Proof. One must check exactness in the categorical sense, but this is not more difficult than for Abelian groups.

The two short exact sequences give rise to a pair of long exact sequences

$$
\begin{aligned}
\ldots & \rightarrow H_{G}^{k+1}\left(X, X^{G} ; \tilde{\omega}_{k}\left(S^{n}\right)\right) \stackrel{p^{*}}{\rightarrow} H_{G}^{k+1}\left(X, X^{G} ; p \tilde{\omega}_{k}\left(S^{n}\right)\right) \\
& \rightarrow H_{G}^{k+2}\left(X, X^{G} ;\left(\tilde{\omega}_{k}\left(S^{n}\right)\right)_{p}\right) \rightarrow \cdots \\
\ldots & \rightarrow H_{G}^{k+1}\left(X, X^{G} ; p \tilde{\omega}_{k}\left(S^{n}\right)\right) \stackrel{i^{*}}{\rightarrow} H_{G}^{k+1}\left(X, X^{G} ; \tilde{\omega}_{k}\left(S^{n}\right)\right) \\
& \rightarrow H_{G}^{k+1}\left(X, X^{G} ; \tilde{\omega}_{k}\left(S^{n}\right) \otimes Z_{p}\right) \rightarrow \cdots .
\end{aligned}
$$

The composition

$$
\begin{aligned}
H_{G}^{k+1}\left(X, X^{G} ; \tilde{\omega}_{k}\left(S^{n}\right)\right) & \stackrel{p^{*}}{\rightarrow} H_{G}^{k+1}\left(X, X^{G} ; p \tilde{\omega}_{k}\left(S^{n}\right)\right) \\
& \stackrel{i^{*}}{\rightarrow} H_{G}^{k+1}\left(X, X^{G} ; \tilde{\omega}_{k}\left(S^{n}\right)\right)
\end{aligned}
$$

is multiplication by $p$ on $H_{G}^{k+1}\left(X, X^{G} ; \tilde{\omega}_{k}\left(S^{n}\right)\right)$. In order for $H_{G}^{k+1}\left(X, X^{G}\right.$; $\left.\tilde{\omega}_{k}\left(S^{n}\right)\right)$ to be of finite order prime to $p$, it suffices, from the above sequences, 
that

$$
H_{G}^{k+2}\left(X, X^{G} ;\left(\tilde{\omega}_{k}\left(S^{n}\right)\right)_{p}\right)=0=H_{G}^{k+1}\left(X, X^{G} ; \tilde{\omega}_{k}\left(S^{n}\right) \otimes Z_{p}\right) .
$$

This brings us to the following lemma.

LEMMA 7. $H_{G}^{j}\left(X, X^{G} ;\left(\tilde{\omega}_{k}\left(S^{n}\right)\right)_{p}\right)=0=H_{G}^{j}\left(X, X^{G} ; \tilde{\omega}_{k}\left(S^{n}\right) \otimes Z_{p}\right)$ for $j>$ $k+1$.

Proof. Consider the following sequence of $G$-spaces:

$$
X=X_{0} \supseteq X_{1} \supseteq \cdots \supseteq X_{r}=X^{G},
$$

where $X_{i}$ is the set of all points in $X$ whose isotropy subgroup has rank $i$ or greater.

We prove this lemma by induction on $i$. From the triple $\left(X_{i-1}, X_{i}, X^{G}\right)$, and assuming the lemma for the pair $\left(X_{i}, X^{G}\right)$, the inductive step will follow if we show the lemma to be true for the pair $\left(X_{i-1}, X_{i}\right)$. When $i=r$ note that this also resolves the initial step of the induction.

First of all, it is easy to check that for any coefficient system $M$,

$$
\operatorname{Hom}\left(C_{*}\left(X_{i-1}, X_{i} ; Z\right), M\right) \approx \bigoplus \operatorname{Hom}_{Z[G / H]}\left(C_{*}\left(X^{H}, X_{1}^{H} ; Z\right), M(G / H)\right) \text {, }
$$

with the sum taken over $\{H \leqslant G \mid H$ has rank $i-1$ in $G\}$. This uses, for instance, the characterisation of equivariant maps $G / H \rightarrow G / K$ given in [3, p. 1.8]. For the particular coefficient systems above, one has

$$
\begin{aligned}
H_{G}^{j}\left(X_{i-1}, X_{i} ;\left(\tilde{\omega}_{k}\left(S^{n}\right)\right)_{p}\right) \\
\quad=\bigoplus H^{j}\left(\operatorname{Hom}_{Z[G / H]}\left(C_{*}\left(X^{H}, X_{1}^{H} ; Z\right), \pi_{k}\left(S^{H}\right)_{p}\right)\right)
\end{aligned}
$$

and

$$
\begin{aligned}
H_{G}^{j}\left(X_{i-1}, X_{i} ; \tilde{\omega}_{k}\left(S^{n}\right) \otimes Z_{p}\right) \\
\quad=\bigoplus H^{j}\left(\operatorname{Hom}_{Z[G / H]}\left(C_{*}\left(X^{H}, X_{1}^{H} ; Z\right), \pi_{k}\left(S^{H}\right) \otimes Z_{p}\right)\right)
\end{aligned}
$$

with the sums over $\{H \leqslant G \mid H$ has rank $i-1$ in $G\}$. It will be shown that each of the summands above is zero. First, as in Lemma 5 and because $C_{*}\left(X^{H}, X_{1}^{H} ; Z\right)$ is a free $Z[G / H]$ complex, $\operatorname{Hom}_{Z[G / H]}\left(C_{*}\left(X^{H}, X_{1}^{H} ; Z\right), Z_{p}\right)$ is chain isomorphic to $\operatorname{Hom}_{Z_{p}[G / H]}\left(C_{*}\left(X^{H}, X_{1}^{H} ; Z_{p}\right), Z_{p}\right)$. The fact that this latter complex is acyclic in dimensions $j$ will follow. The assertion about the summands above, then, is immediate by an induction on the order of the $p$-groups $\pi_{k}\left(S^{H}\right)_{p}$ and $\pi_{k}\left(S^{H}\right) \otimes Z_{p}$, just as in the proof of Lemma 5. Note that we may as well assume $k \geqslant n(H)$.

LEMMA 8. $H^{j}\left(\operatorname{Hom}_{Z_{p}[G / H]}\left(C_{*}\left(X^{H}, X_{1}^{H} ; Z_{p}\right), Z_{p}\right)\right)=0$ for $j>k+1$.

Proof. If $m$ is the cellular dimension of $X^{H}$ we have the sequence, 


$$
\begin{aligned}
0 & \rightarrow C_{m}\left(X^{H}, X_{1}^{H} ; Z_{p}\right) \rightarrow \cdots \rightarrow C_{k+1}\left(X^{H}, X_{1}^{H} ; Z_{p}\right) \\
& \rightarrow C_{k}\left(X^{H}, X_{1}^{H} ; Z_{p}\right) \rightarrow \frac{C_{k}\left(X^{H}, X_{1}^{H} ; Z_{p}\right)}{B_{k}\left(X^{H}, X_{1}^{H} ; Z_{p}\right)} \rightarrow 0
\end{aligned}
$$

where $B_{k}\left(X^{H}, X_{1}^{H} ; Z_{p}\right)$ are the relative $k$-boundaries. If this sequence were exact then the $Z_{p}[G / H]$-module $C_{k}\left(X^{H}, X_{1}^{H} ; Z_{p}\right) / B_{k}\left(X^{H}, X_{1}^{H} ; Z_{p}\right)$ would have finite projective dimension and so be projective over $Z_{p}[G / H]$. The result is then immediate since the cohomology we seek is just

$$
\operatorname{Ext}_{Z_{p}[G / H]}^{j-k}\left(C_{k}\left(X^{H}, X_{1}^{H} ; Z_{p}\right) / B_{k}\left(X^{H}, X_{1}^{H} ; Z_{p}\right) ; Z_{p}\right)=0 .
$$

So, it suffices to show that $H_{j}\left(X^{H}, X_{1}^{H} ; Z_{p}\right)=0$, if $j>n(H)$, to which end we now devote our attention.

In what follows, all homology will be with $Z_{p}$-coefficients, suppressed. The argument is a double induction on the rank of $H$ and on the number of entries in the union $X_{1}^{H}=\cup X^{K}$, running over $\left\{K<G \mid K / H=Z_{p}\right\}$. To begin with, the assertion is certainly true if $H=G$ or the union is empty. Now suppose there exists some $M_{0} \geqslant H$ such that $M_{0} / H=Z_{p}$ with $n\left(M_{0}\right)<$ $n(H)$. Let $A_{M_{0}}=\left\{M>H \mid M / H=Z_{p}, M \neq M_{0}\right\}$. Considering the triple $\left(X^{H}, X_{1}^{H}, \cup X^{M}\right)$, where the union runs over $A_{M_{0}}$, one has

$$
\ldots \rightarrow H_{j}\left(X^{H}, \bigcup X^{M}\right) \rightarrow H_{j}\left(X^{H}, X_{1}^{H}\right) \rightarrow H_{j-1}\left(X_{1}^{H}, \bigcup X^{M}\right) \rightarrow \ldots
$$

The left-hand group is zero by induction because the union has fewer terms. Concentrating on the right-hand group, and by excision,

$$
H_{j-1}\left(X_{1}^{H}, \bigcup X^{M}\right)=H_{j-1}\left(X^{M_{0}}, \bigcup\left(X^{M} \cap X^{M_{0}}\right)\right) \text {, }
$$

with unions over $A_{M_{0}}$. Now let $K$ be any subgroup of $G$ containing $M_{0}$ such that $K / M_{0}=Z_{p}$. Let $\bar{g}_{0}=g_{0}+M_{0}$ generate $K / M_{0}$ and consider $M_{1}=$ $\left(H, g_{0}\right)$, the subgroup generated by $H$ and $g_{0}$. Clearly $M_{1} \neq M_{0}$, and $X^{M_{1}} \cap$ $X^{M_{0}}=X^{K}$, since $K=\left(M_{0}, g_{0}\right)$. So $\left(X^{M_{0}}, \cup\left(X^{M} \cap X^{M_{0}}\right)\right)=\left(X^{M_{0}}, X_{1}^{M_{0}}\right)$, with union over $A_{M_{0}}$. Since $j-1>n\left(M_{0}\right)$ the inductive assumption applies and $H_{j-1}\left(X^{M_{0}}, X_{1}^{M_{0}}\right)=0$, so that $H_{j}\left(X^{H}, X_{1}^{H}\right)=0$.

Now if no $M>H$ with $M / H=Z_{p}$ has the property $n(M)<n(H)$, we claim this forces $n(H)=n(G)$. In order to see this, we examine the Borel formula for the $G / M$ action on $X^{M}$ and the $G / H$ action on $X^{H}$, where $M / H=Z_{p}$. These state that

$$
\begin{aligned}
n(M)-n(G) & =\sum(n(K)-n(G)) \quad \text { and } \\
n(H)-n(G) & =\sum\left(n\left(K^{\prime}\right)-n(G)\right)
\end{aligned}
$$

where each of the sums is over $\left\{K>M\right.$ (resp. $K^{\prime}>H$ )|K (resp. $K^{\prime}$ ) has rank $r-1$ \}. Now $n(M)=n(H)$ implies the two sums are equal, and after 
cancellation of common terms, one has

$$
0=\sum\left(n\left(K^{\prime}\right)-n(G)\right)
$$

where this sum runs over $\left\{K^{\prime} \geqslant H \mid K^{\prime} \succcurlyeq M\right.$ and $K^{\prime}$ has rank $\left.r-1\right\}$. Thus for all $K^{\prime}$ appearing in equation (2), $n\left(K^{\prime}\right)=n(G)$. Let $K^{\prime}$ be any group appearing in the first equation of (1). $K^{\prime}$ has corank 1 in $G$, so let $\bar{g}=g+K^{\prime}$ generate $G / K^{\prime}$. Considering $M_{1}=(H, g)$, it is clear that $K^{\prime} \ngtr M_{1}$. So, $K^{\prime}$ appears in equation (2) for $M=M_{1}$. Hence $n\left(K^{\prime}\right)=n(G)$ and $K^{\prime}$ arbitrary shows $n(H)=n(G)$. Now $M / H$ acts on $X^{H}$ and if $n(M)=n(H)$, the inclusion $X^{M} \rightarrow X^{H}$ induces a $Z_{p}$-homology isomorphism. This fact and an obvious induction and relative Mayer-Vietoris argument shows that $\left(X^{H}, X_{1}^{H}\right)$ is an acyclic pair (if $n(H)=n(G)$ ). So $H_{j}\left(X^{H}, X_{1}^{H}\right)=0$ if $j>$ $n(H)$.

This completes the discussion of the obstructions to producing the map $\phi$.

4. We now suppose we have a $G$-map $\phi: X \rightarrow S^{n}$ such that $\phi^{G}: X^{G} \rightarrow S^{G}$ induces a $Z_{p}$-homology isomorphism. It will be seen that, in fact, for $H<G$, $H \neq 0, \phi^{H}: X^{H} \rightarrow S^{H}$ induces a $Z_{p}$-homology isomorphism and an epimorphism if $H=0$. The vital fact is the following lemma.

LEMMA 9. Suppose $X$ is a finite $C W$-complex with $a G=Z_{p}$ cellular action such that $X^{G}$ is a $Z_{p}$-homology $r$-sphere. Also suppose there is an $n>0$ so that $\tilde{H}_{i}\left(X ; Z_{p}\right)=0, i \neq n$, and let $\phi: X \rightarrow S^{n}$ be G-equivariant with $\phi^{G}: X^{G} \rightarrow$ $\left(S^{n}\right)^{G}$ inducing a $Z_{p}$-homology isomorphism.

Then $\phi$ induces a $Z_{p}$-homology epimorphism

$$
\phi_{*}: H_{n}\left(X ; Z_{p}\right) \rightarrow H_{n}\left(S^{n} ; Z_{p}\right)
$$

Proof. We will use the notation of [2, Chapter 3].

If $r=n$, it is well known that $i:\left(S^{n}\right)^{G} \rightarrow S^{n}$ induces a $Z_{p}$-homology isomorphism. The following commutative diagram $\left(Z_{p}\right.$-coefficients throughout)

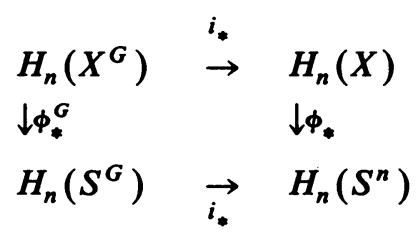

shows that $\phi_{*}$ must be an epimorphism.

Now suppose $r<n$ and consider the standard Smith diagram $\left(Z_{p}\right.$ coefficients) 


$$
\begin{aligned}
& \rightarrow H_{r+1}(X) \rightarrow H_{r+1}^{o}(X) \rightarrow H_{r}^{\tau}(X) \oplus H_{r}\left(X^{G}\right) \rightarrow H_{r}(X) \rightarrow \\
& \downarrow \phi_{*} \quad \downarrow \phi_{*}^{0} \quad \downarrow \phi_{*}^{*} \quad \downarrow \phi_{*}^{G} \quad \downarrow \phi_{*} \\
& \rightarrow H_{r+1}\left(S^{n}\right) \rightarrow H_{r+1}^{o}\left(S^{n}\right) \rightarrow H_{r}^{\tau}\left(S^{n}\right) \oplus H_{r}\left(S^{G}\right) \rightarrow H_{r}\left(S^{n}\right) \rightarrow
\end{aligned}
$$

Because rank $H_{r}^{\tau}\left(S^{n}\right)+\Sigma_{i>r} \operatorname{rank} H_{i}\left(S^{\sigma}\right)<\Sigma_{i>r} \operatorname{rank} H_{i}\left(S^{n}\right)$ we have $H_{r}^{\tau}\left(S^{n}\right)=0$. The same inequality with $X$ replacing $S^{n}$ and $r$ replaced by $m$ $(>n)$ shows that $(i>n) H_{i}^{o}(X)=0=H_{i}^{\tau}(X)$. Similarly for $S^{n}$. Some other, easily established facts we will need (see [2, Chapter 3]) are that $H_{n}^{o}\left(S^{n}\right)=$ $H_{n}\left(S / G, S^{G}\right)=Z_{p}$ if $r=n-1$ and for $r<n-2, H_{n}^{o}\left(S^{n}\right)=Z_{p}=$ $H_{n-1}^{o}\left(S^{n}\right)$.

Now suppose $r=n-1$ (and $p=2$, necessarily). Then the Smith diagram becomes, after simplifying,

$$
\begin{aligned}
0 & \rightarrow H_{n}^{\tau}(X) \rightarrow H_{n}(X) & \rightarrow H_{n}^{o}(X) \rightarrow H_{n-1}^{\tau}(X) \oplus H_{n-1}\left(X^{G}\right) & \rightarrow 0 \\
& \downarrow \phi_{*}^{\tau} \quad \downarrow \phi_{*} \quad \downarrow \phi_{*}^{0} & \downarrow \phi_{*}^{G} & \\
0 \rightarrow & H_{n}^{\tau}\left(S^{n}\right) \rightarrow H_{n}\left(S^{n}\right) \rightarrow H_{n}^{o}\left(S^{n}\right) \rightarrow & H_{n-1}\left(S^{G}\right) & \rightarrow 0
\end{aligned}
$$

where some of the facts listed above have been used. A diagram chase verifies that $\phi_{*}^{o}: H_{n}^{o}(X) \rightarrow H_{n}^{o}\left(S^{n}\right)$ is an epimorphism. Writing down the dual diagram to the one above (i.e. with the roles of $\sigma$ and $\tau$ reversed), one has

$$
\begin{aligned}
& 0 \rightarrow H_{n}^{o}(X) \rightarrow H_{n}(X) \rightarrow H_{n}^{\tau}(X) \rightarrow H_{n-1}^{o}(X) \oplus H_{n-1}\left(X^{G}\right) \rightarrow 0 \\
& \downarrow \phi_{*}^{0} \quad \downarrow \phi_{*} \quad \downarrow \phi_{*}^{\tau} \quad \downarrow \phi_{*}^{0} \quad \downarrow \phi_{*}^{G} \\
& 0 \rightarrow H_{n}^{\sigma}\left(S^{n}\right) \underset{i_{*}}{\rightarrow} H_{n}\left(S^{n}\right) \rightarrow H_{n}^{\tau}\left(S^{n}\right) \rightarrow H_{n-1}^{o}\left(S^{n}\right) \oplus H_{n-1}\left(S^{G}\right) \rightarrow 0
\end{aligned}
$$

Since $H_{n}^{o}\left(S^{n}\right)=Z_{p}, i_{*}$ is an isomorphism; this and the surjectivity of $\phi_{*}^{\sigma}$ are enough to ensure that $\phi_{*}$ is onto.

Now if $r<n-2$, the Smith diagram (3) simplifies to

$$
\begin{array}{cccc}
0 \rightarrow \underset{r+1}{H_{\phi_{*}^{o}}^{o}(X)} & \rightarrow & H_{r}^{\tau}(X) \oplus H_{r}\left(X^{G}\right) & \rightarrow 0 \\
0 \rightarrow H_{r+1}^{o}\left(S^{n}\right) & \rightarrow & H_{r}\left(S^{G}\right) & \rightarrow 0
\end{array}
$$

It follows that $\phi_{*}^{0}$ is an epimorphism and, from the dual of this diagram (in the sense above), $\phi_{*}^{\tau}$ is also an epimorphism.

Now consider

$$
\begin{aligned}
& H_{r+2}(X) \rightarrow H_{r+2}^{o}(X) \rightarrow H_{r+1}^{\tau}(X) \oplus H_{r+1}\left(X^{G}\right) \rightarrow H_{r+1}(X) \\
& \downarrow \phi_{*} \quad \downarrow \phi_{*}^{0} \quad \downarrow \phi_{*}^{*} \quad \downarrow \phi_{*}^{G} \quad \downarrow \phi_{*} \\
& H_{r+2}\left(S^{n}\right) \rightarrow H_{r+2}^{o}\left(S^{n}\right) \rightarrow H_{r+1}^{\tau}\left(S^{n}\right) \oplus H_{r+1}\left(S^{G}\right) \rightarrow H_{r+1}\left(S^{n}\right)
\end{aligned}
$$


Since $H_{r+1}\left(X^{G}\right)=0$, this simplifies to

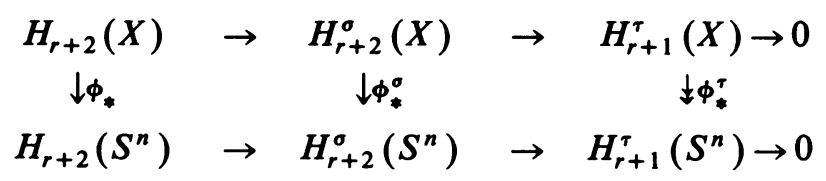

If $r+2<n$, we immediately get that $\phi_{*}^{\sigma}: H_{r+2}^{\sigma}(X) \rightarrow H_{r+2}^{\sigma}\left(S^{n}\right)$ is an epimorphism and, by considering the dual, we see that $\phi_{*}^{\tau}: H_{r+2}^{\tau}(X) \rightarrow H_{r+2}^{\tau}\left(S^{n}\right)$ is an epimorphism. We continue in this way (i.e. deducing that various maps are onto) until we arrive at

$$
\begin{aligned}
& 0 \rightarrow H_{n}^{\sigma}(X) \quad \rightarrow \quad H_{n}(X) \quad \rightarrow \quad H_{n}^{\tau}(X) \quad \rightarrow \quad H_{n-1}^{\sigma}(X) \rightarrow 0 \\
& \downarrow \phi_{*}^{0} \quad \downarrow \phi_{*} \quad \downarrow \phi_{*}^{\tau} \quad \downarrow \phi_{*}^{\text {q }}
\end{aligned}
$$

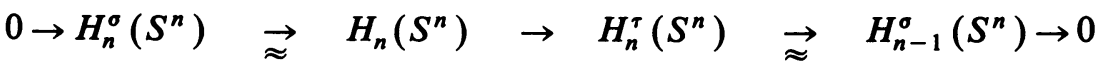

The isomorphisms have already been noted, and so it follows that $\phi_{*}^{\tau}$ : $H_{n}^{\tau}(X) \rightarrow H_{n}^{\tau}\left(S^{n}\right)$ is surjective. Now we write down the diagram (dual to this last one)

$$
\begin{aligned}
& 0 \rightarrow H_{n}^{\tau}(X) \quad \rightarrow \quad H_{n}(X) \quad \rightarrow \quad H_{n}^{\sigma}(X) \quad \rightarrow \quad H_{n-1}^{\tau}(X) \rightarrow 0 \\
& \downarrow \phi_{*}^{\tau} \quad \downarrow \phi_{*} \quad \downarrow \phi_{*}^{0} \quad \downarrow \phi_{*}^{\tau}
\end{aligned}
$$

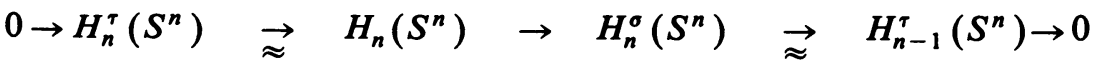

The surjectivity of $\phi_{*}^{\tau}: H_{n}^{\tau}(X) \rightarrow H_{n}^{\tau}\left(S^{n}\right)$ and the isomorphism $H_{n}^{\tau}\left(S^{n}\right) \rightarrow$ $H_{n}\left(S^{n}\right)$ (in case $r<n-2$ ) shows that $\phi_{*}$ is an epimorphism.

Applying this lemma to the action of $G / H$ on $X^{H}$ where $H$ has corank 1 in $G$, one gets that $\phi^{H}: X^{H} \rightarrow S^{H}$ induces a $Z_{p}$-homology isomorphism. Continuing in this way, one deduces that if $H \triangleleft G, H \neq 0$, then $\phi^{H}$ : $X^{H} \rightarrow S^{H}$ is a $Z_{p}$-homology isomorphism while $\phi: X \rightarrow S^{n}$ is a $Z_{p}$-homology epimorphism.

\section{REFERENCES}

1. A. Borel, Seminar on transformation groups, Ann. of Math. Studies, no. 46, Princeton Univ. Press, Princeton, N. J., 1960.

2. G. Bredon, Introduction to compact transformation groups, Academic Press, New York, 1972.

3. __ Equivariant cohomology theories, Lecture Notes in Math., vol. 34, Springer-Verlag, Berlin and New York, 1967.

4. H. Cartan and S. Eilenberg, Homological algebra, Princeton Math. Series, no. 19, Princeton Univ. Press, Princeton, N. J., 1956.

5. R. Oliver, Fixed-point sets of group actions on finite acyclic complexes, Comment. Math. Helv. 50 (1975), 155-177.

6. D. S. Rim, Modules over finite groups, Ann. of Math. (2) 69 (1959), 700-712.

7. J.-P. Serre, Représentations linéaires des groupes finis, 2nd ed., Hermann, Paris, 1971.

8. E. Spanier, Algebraic topology, McGraw-Hill, New York, 1966.

Department of Mathematics, University of Texas, Austin, Texas 78712 